Supporting Information

for

\title{
Stereoselective Construction of Spiro-fused Tricyclic Frameworks by Sequential Reaction of Enynes, Imines, and Diazoalkenes with Rh(I) and $\mathrm{Rh}(\mathrm{II})$ Catalysts
}

Yoshio Hato, ${ }^{[\mathrm{a}, \mathrm{b}]}$ Yoshihiro Oonishi, ${ }^{[\mathrm{a}]}$ Yasunori Yamamoto, ${ }^{[\mathrm{c}]}$ Kiyohiko Nakajima, ${ }^{[\mathrm{d}]}$ and Yoshihiro Sato*[a]

${ }^{[\mathrm{a}]}$ Faculty of Pharmaceutical Sciences, Hokkaido University, Sapporo 060-0812, Japan

${ }^{\left[{ }^{b}\right]}$ Shionogi Innovation Center for Drug Discovery, Shionogi \& Co., Ltd., Sapporo 001-0021, Japan

${ }^{[\mathrm{c}]}$ Division of Chemical Process Engineering and Frontier Chemistry Center, Faculty of Engineering, Hokkaido University, Sapporo 060-8628, Japan

${ }^{[\mathrm{d}]}$ Department of Chemistry, Aichi University of Education, Kariya 448-8542, Japan

\section{Table of Contents}

Structural Determination of Compound 7 by X-ray Analysis

$1-3$

${ }^{1} \mathrm{H}$ NMR and ${ }^{13} \mathrm{C}$ NMR spectra 


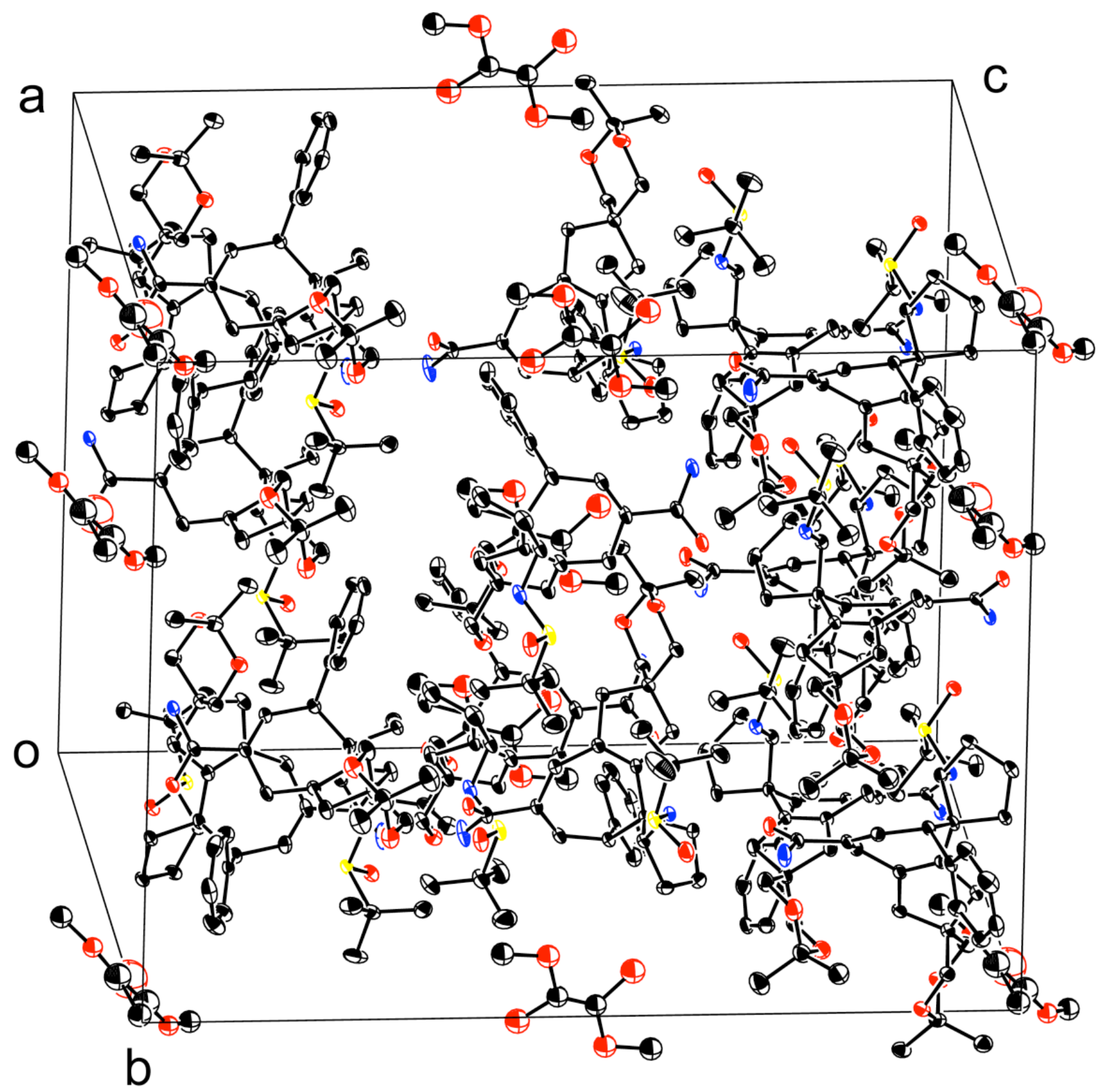

Figure S1. An ORTEP Drawing of 7. The ellipsoid contour percent probability level is $30 \%$. 
Empirical Formula

Formula Weight

Crystal Color, Habit

Crystal Dimensions

Crystal System

Lattice Type

Lattice Parameters

Space Group

$Z$ value

Dcalc

$\mathrm{F}_{000}$

$\mu(\mathrm{CuK} \alpha)$

Diffractometer

Radiation

Voltage, Current

Temperature

Detector Aperture

Detector Position

Pixel Size

$2 \theta \max$

No. of Reflections Measured

Corrections

Structure Solution

Refinement

Function Minimized
$\mathrm{C}_{31} \mathrm{H}_{42} \mathrm{~N}_{2} \mathrm{O}_{4.67} \mathrm{~S}$

549.46

colorless, chip

0.840 X 0.550 X $0.020 \mathrm{~mm}$

monoclinic

C-centered

$\mathrm{a}=22.508(3) \AA$

$\mathrm{b}=15.8459(17) \AA$

$\mathrm{c}=26.254(4) \AA$

$\mathrm{g}=93.149(4)^{\mathrm{o}}$

$\mathrm{V}=9349.6(19) \AA^{3}$

C2 (\#5)

12

$1.171 \mathrm{~g} / \mathrm{cm}^{3}$

3544.32

$12.264 \mathrm{~cm}^{-1}$

XtaLAB P200

$\operatorname{CuK} \alpha(\lambda=1.54187 \AA)$

multi-layer mirror monochromated

$40 \mathrm{kV}, 30 \mathrm{~mA}$

$-173.0^{\circ} \mathrm{C}$

$83.8 \times 70.0 \mathrm{~mm}$

$35.15 \mathrm{~mm}$

$0.172 \mathrm{~mm}$

$150.9^{\circ}$

Total: 64326

Unique: $18083(\operatorname{Rint}=0.0859)$

Friedel pairs: 8323

Lorentz-polarization

Absorption

(trans. factors: $0.706-0.976$ )

Direct Methods (SIR2004)

Full-matrix least-squares on $\mathrm{F}^{2}$

$\Sigma \mathrm{W}\left(\mathrm{Fo}^{2}-\mathrm{Fc}^{2}\right)^{2}$ 
Least Squares Weights

$2 \theta$ max cutoff

Anomalous Dispersion

No. Observations (All reflections)

No. Variables

Reflection/Parameter Ratio

Residuals: R1 (I>2.00б(I))

Residuals: R (All reflections)

Residuals: wR2 (All reflections)

Goodness of Fit Indicator

Flack Parameter $($ Friedel pairs $=8323)$

Max Shift/Error in Final Cycle

Maximum peak in Final Diff. Map

Minimum peak in Final Diff. Map
$1 /\left[0.0058 \mathrm{Fo}^{2}+1.0000 \sigma\left(\mathrm{Fo}^{2}\right)\right] /\left(4 \mathrm{Fo}^{2}\right)$

$150.9^{\circ}$

All non-hydrogen atoms

18083

1127

16.05

0.0621

0.0771

0.1598

0.713

$-0.009(13)$

0.005

$2.60 \mathrm{e}^{-} / \AA^{3}$

$-1.05 \mathrm{e}^{-} / \AA^{3}$ 

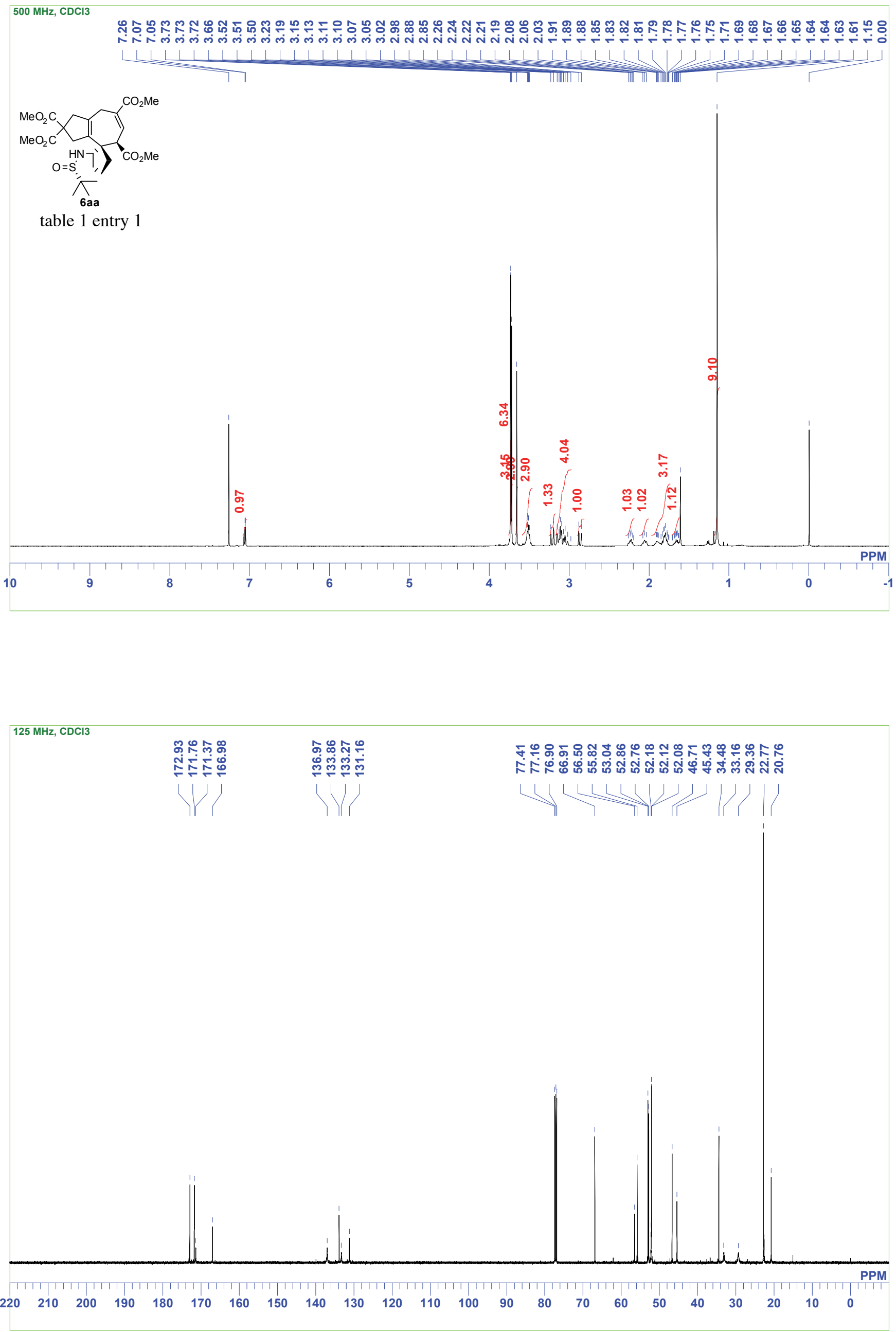

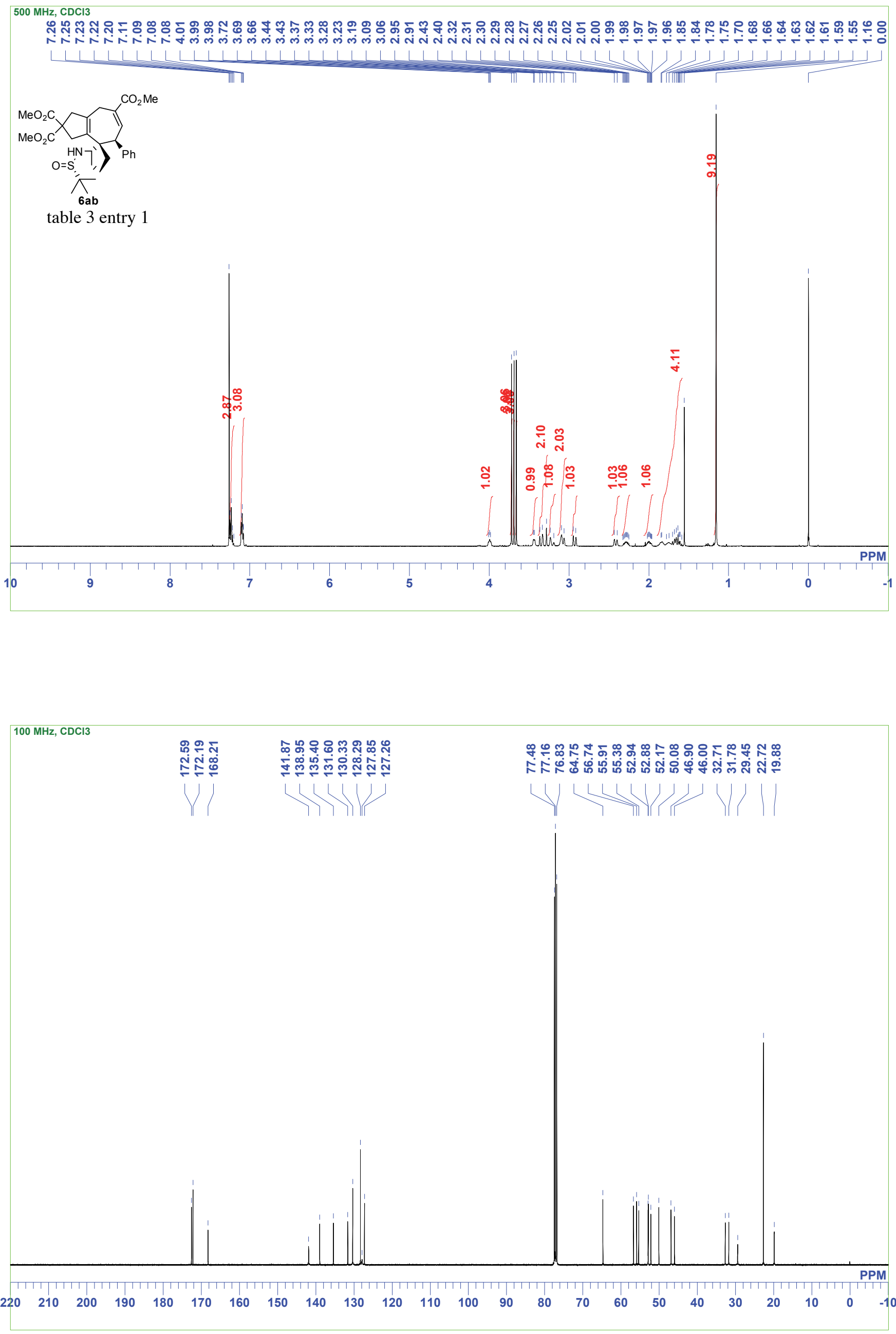

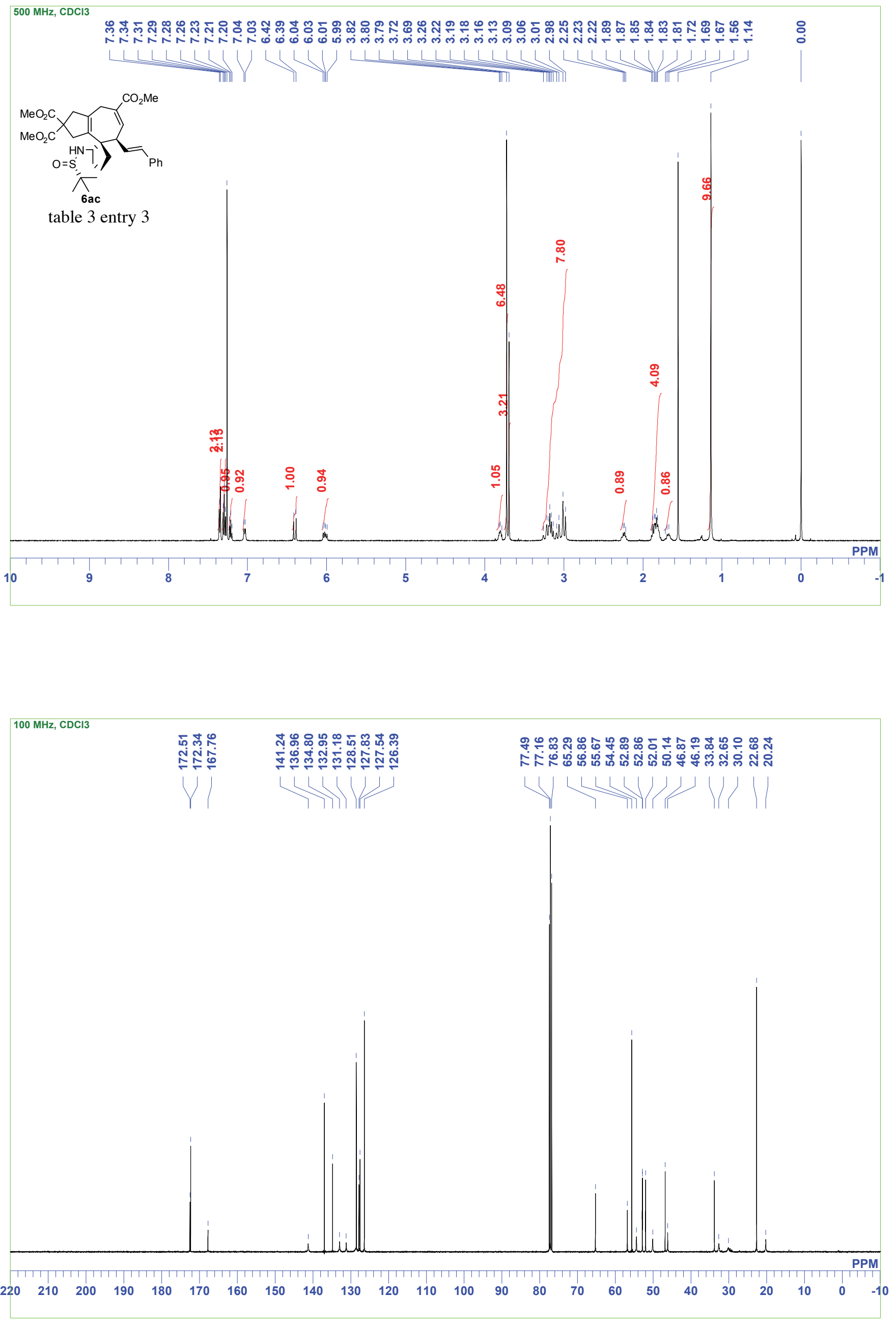

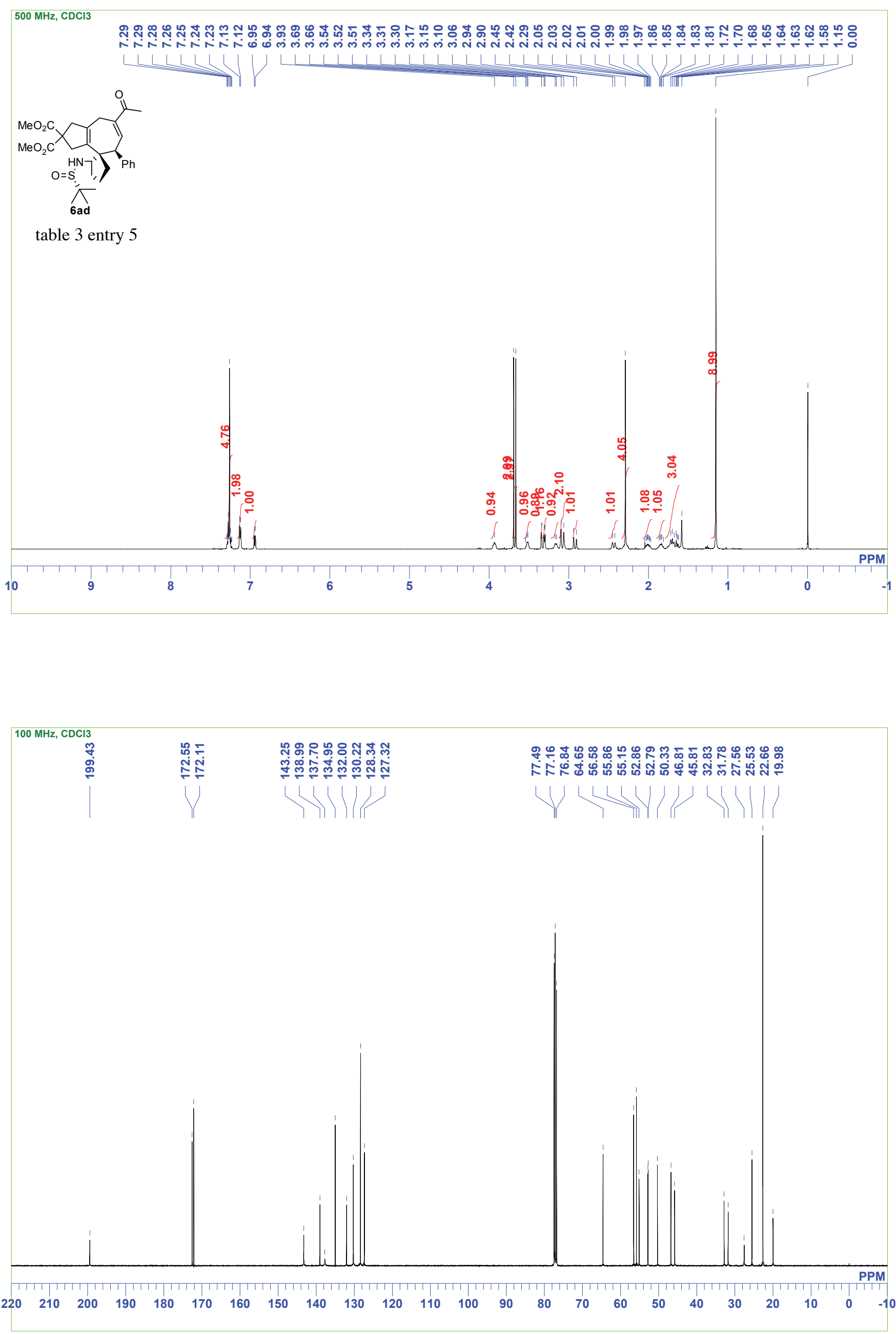


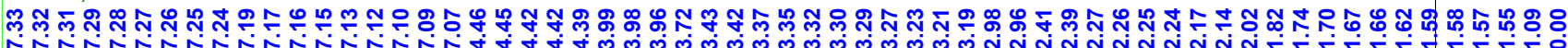
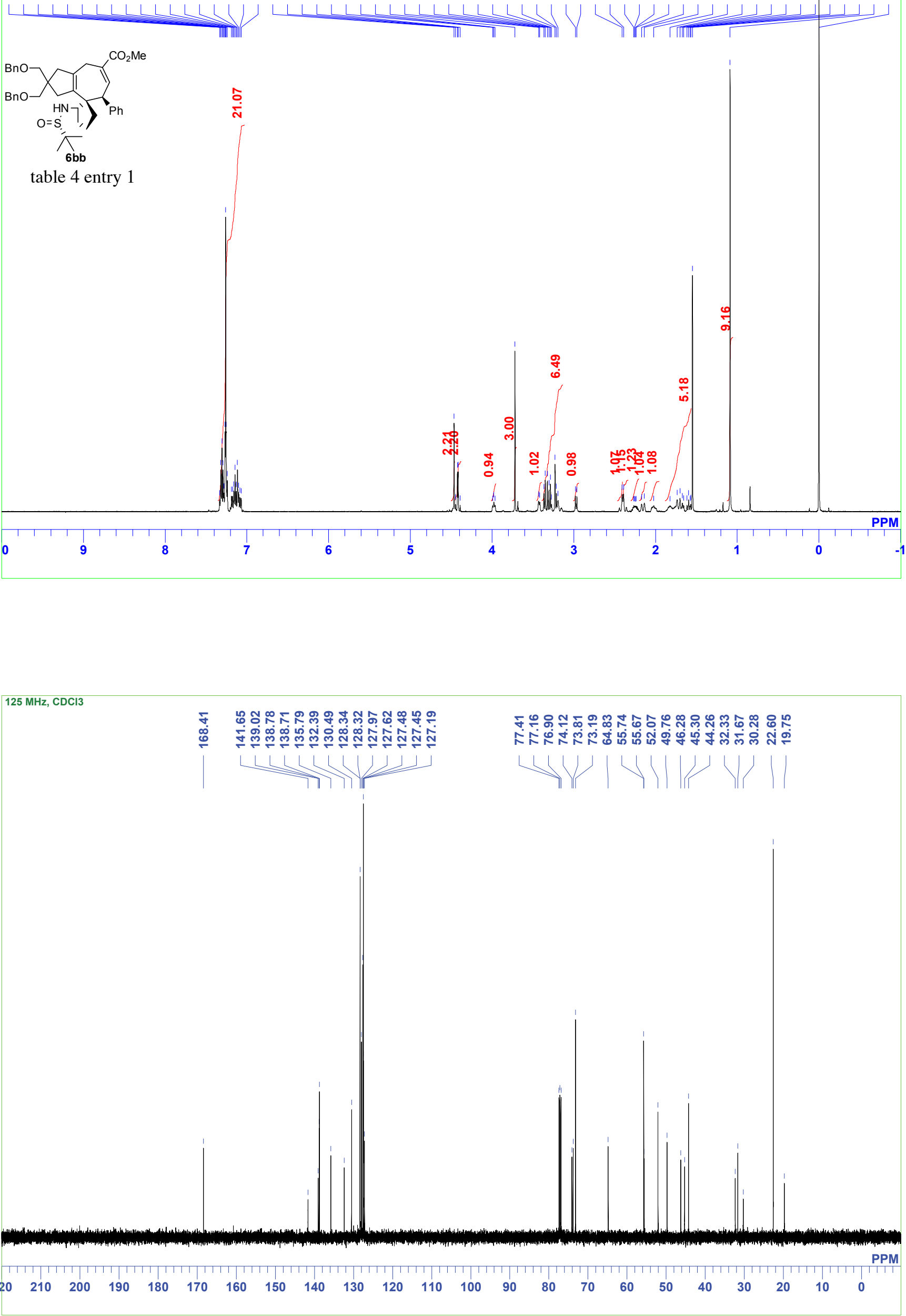


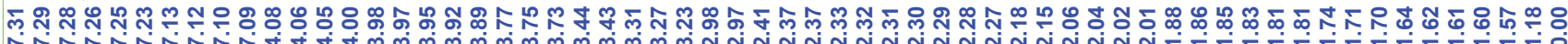
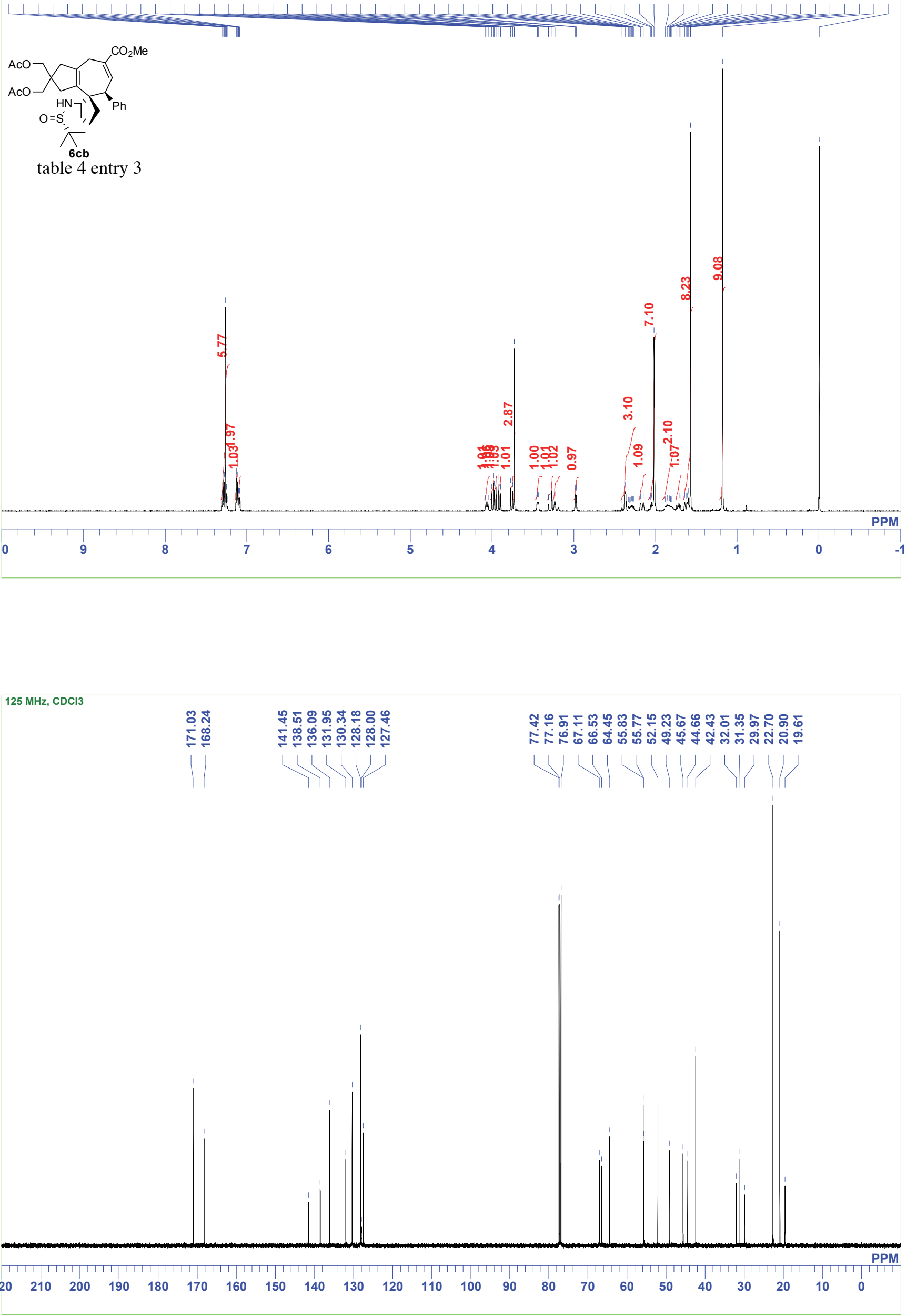

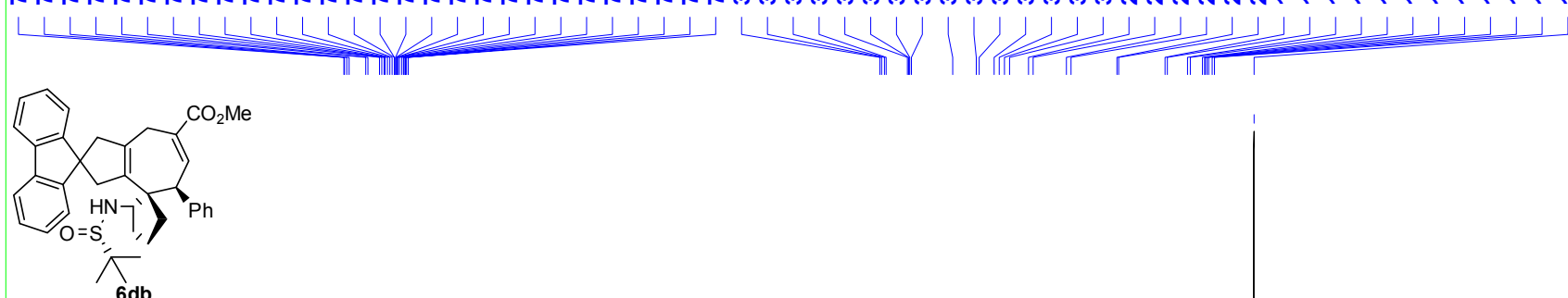

table 4 entry 5

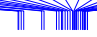

II 11110
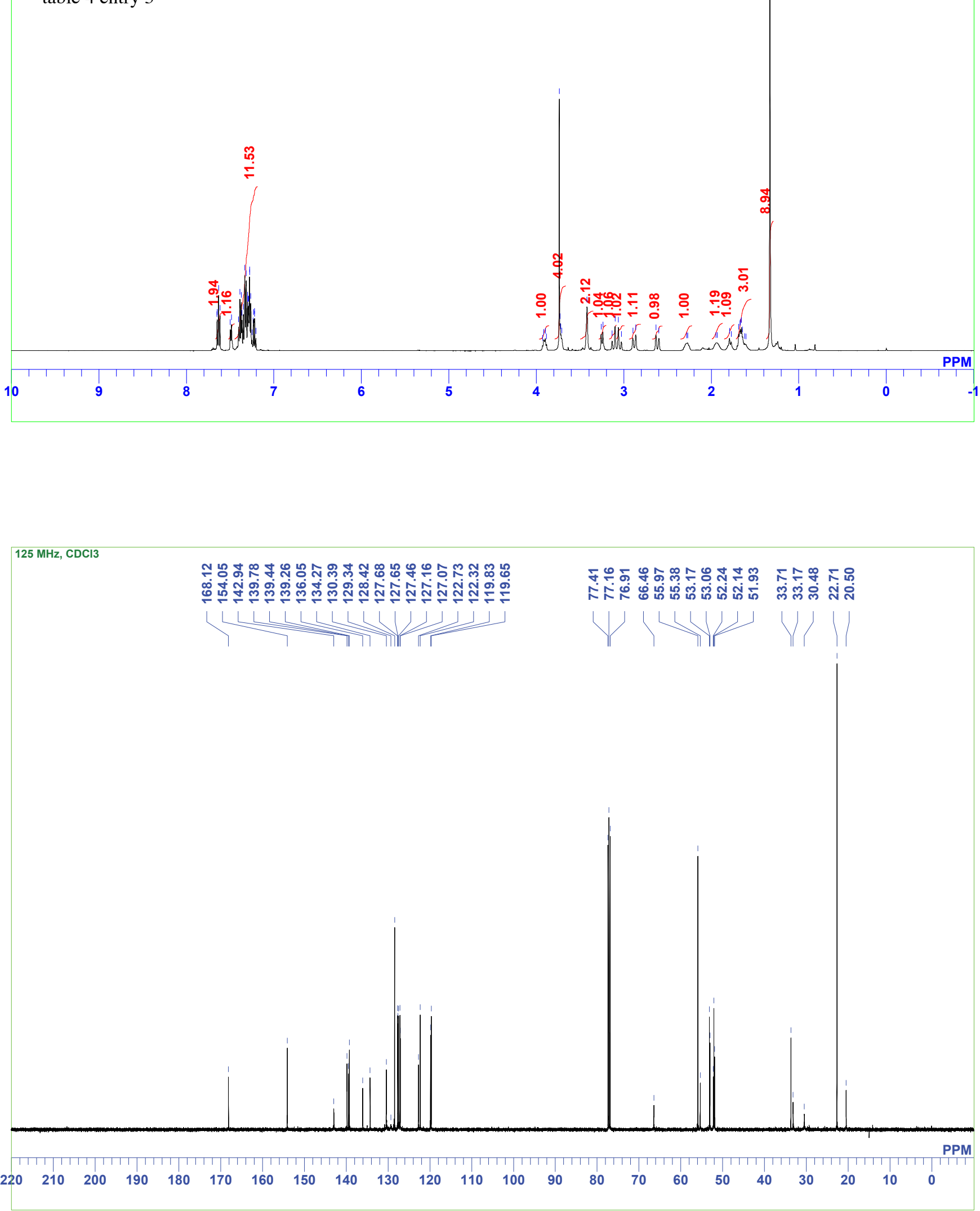


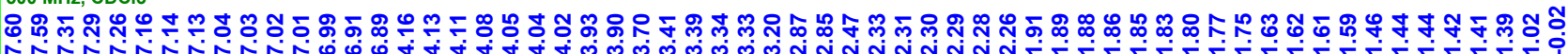
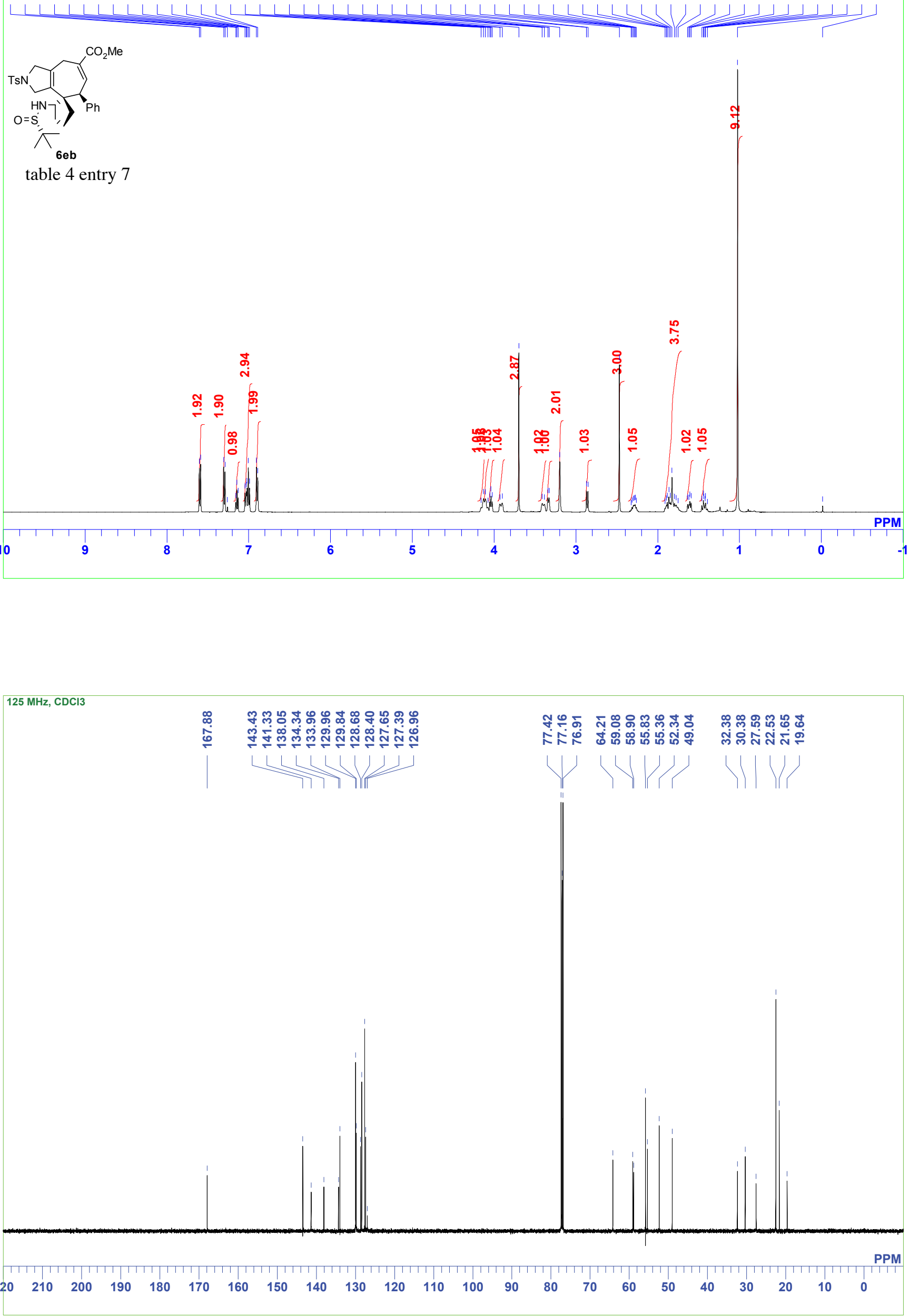


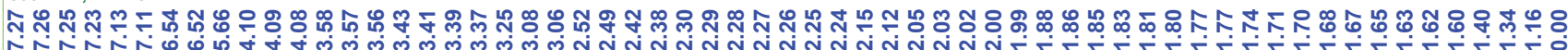
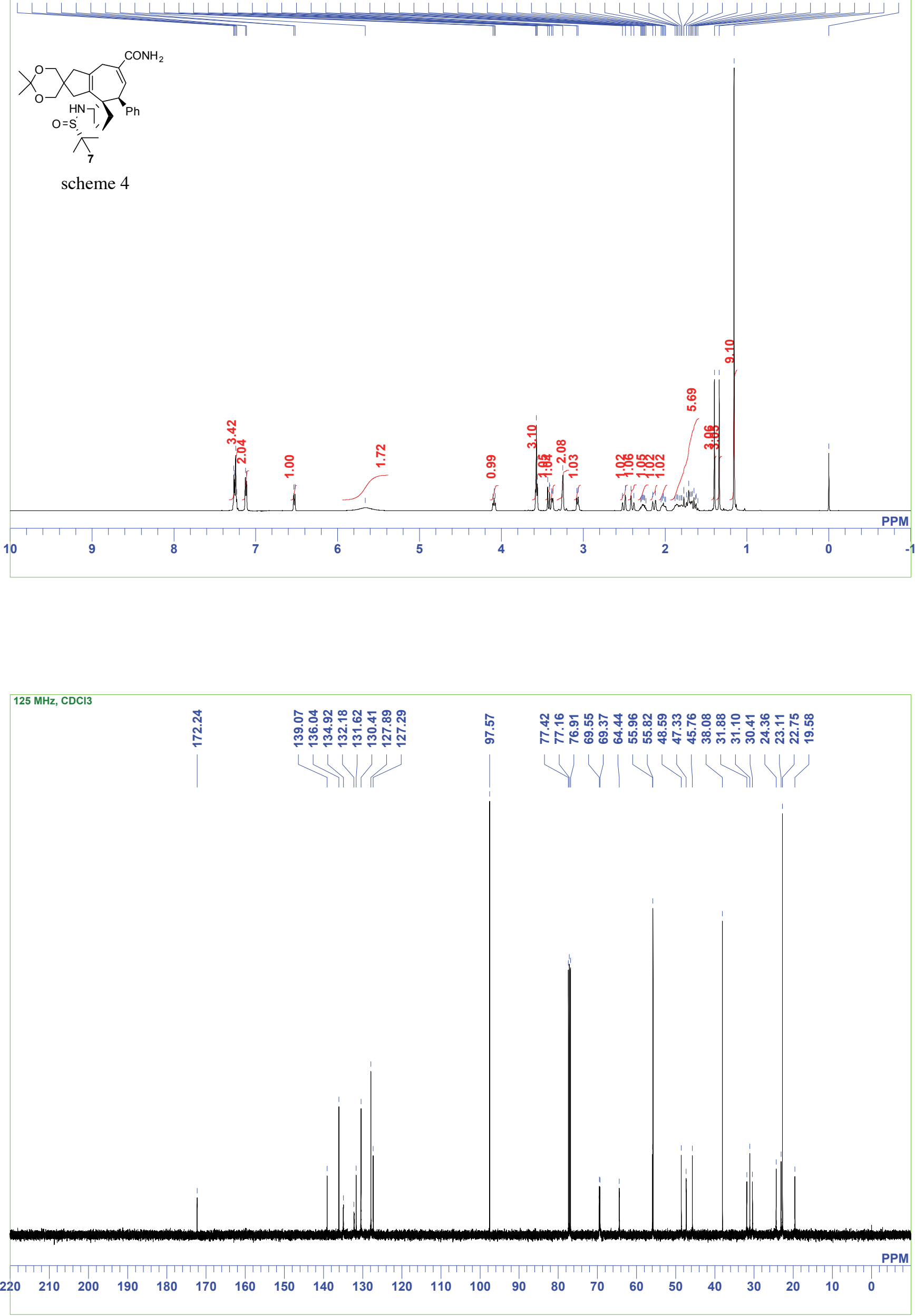